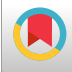

\title{
Predictive Factors for Disability in Patients with Acute and Chronic Headache
}

\author{
Maryam Shaygan ${ }^{1, *}$, Sasan Rahmanian ${ }^{2}$, Farhad Emadi ${ }^{3}$ and Mahnaz Rakhshan ${ }^{2}$ \\ ${ }^{1}$ Community Based Psychiatric Care Research Centre, Faculty of Nursing and Midwifery, Shiraz University of Medical Sciences, Shiraz, Iran \\ ${ }^{2}$ Faculty of Nursing and Midwifery, Shiraz University of Medical Sciences, Shiraz, Iran \\ ${ }^{3}$ Shiraz University of Medical Sciences, Shiraz, Iran \\ "Corresponding author: Faculty of Nursing and Midwifery, Shiraz University of Medical Sciences, P.O. Box. 713451359, Shiraz, Iran. Email: m2620.shaygan@gmail.com
}

Received 2018 December 13; Revised 2019 May 10; Accepted 2019 May 15.

\begin{abstract}
Background: Headache is one of the most common types of pain which is considered among the most disabling of diseases. However, the severity of disability in some headache patients is more than others.

Objectives: The aim of the present study was to compare different types of headache regarding pain-related variables and psychological factors. We also examined the predicting factors of disability in patients with headache.

Methods: This cross-sectional study was performed in 320 patients with various types of headache based on the International Headache Society criteria, which was assessed by neurologists. Data was collected using migraine disability assessment for disability, frequency of headache and for pain intensity, patient health questionnaire for depression, and pain anxiety symptoms scale for pain-related anxiety. ANOVAs and post hoc Tukey's tests were used for comparing various types of headaches regarding pain-related and psychological factors. Regression analyses assessed the relation of pain-related and psychological variables with disability.

Results: Patients with different types of headache revealed no significant differences regarding pain intensity $(\mathrm{P}=0.27)$. Migraine patients showed the most pain frequency and chronicity compared to the other patients $(\mathrm{P}=0.000)$. The levels of disability $(\mathrm{P}=$ $0.000)$, anxiety $(\mathrm{P}=0.000)$ and depressive symptoms $(\mathrm{P}=0.000)$ were also higher in patients with migraine compared to patients suffering from cluster or tension type headache. Pain chronicity $(P=0.01)$, anxiety $(P=0.007)$ and depression $(P=0.002)$ made significant contributions to the explanation of variance in 'disability'.

Conclusions: The findings add further evidence to the relevance of cognitive-behavioral models of pain suggesting an important role for pain-related emotions regarding the consequences of pain (e.g. disability). The current data could help clinicians to decide which factors should be considered for a successful treatment of disability in headache patients.
\end{abstract}

Keywords: Headache, Disability, Chronicity, Depression, Anxiety

\section{Background}

Pain is among the most common reasons for which individuals seek medical attention. It creates personal, familial and social burden which affects various aspects of an individual's life $(1,2)$. According to the definition of International Association for the Study of Pain (IASP), pain is an unpleasant sensory and emotional experience that is associated with actual or potential tissue damage (3). Pain is divided into two categories: acute pain and chronic pain. Acute pain is a type of pain lasting for less than 3 months. Whereas chronic pain is pain that is ongoing and usually lasts longer than six months (4).

Headache is one of the most common types of pain, so that more than 80 percent of people experience headache for at least one day in a year and the primary complaint of 10 - 12 percent of those who refer to the physician is headache (5-8). Headaches are divided into two primary and secondary categories according to the definition of the International Headache Society (HIS). Primary headaches are known as a group of headaches which aren't attributed to a specific illness ground or medical cause. Secondary headaches can be attributed to pathogenic conditions such as infection, malignancy, medication, etc. (5). The most common and prevalent types of primary headaches are migraine, tension type headache and cluster type headache (9). According to the criteria of the International Headache Society, if the headache symptoms last at least 15 days each month for at least 3 months, migraine, tension type and cluster headaches are considered as chronic (9).

Headache can lead to functional disability in the pa- 
tients (10-12), so much that it is the second cause of disability in the world according to recent studies (10). The disability which is due to headache represents the limitation of an activity that a person is able to perform normally. This definition involves a person's perception of his/her abilities. For example, a patient with fear of pain and catastrophizing may consider the situation more threatening than its actual size and estimates his/her ability in dealing with threats less than its actual size. So, he/she has a more negative feeling about his/her self (13). Continuous feeling of disability can provide helplessness, negative mood and depression (14).

It is obvious that the intensity of disability in some patients with headache is much higher than the others and it is not completely known that how migraine attacks or other types of headaches may associate with disability in patients with headache. Some previous studies have shown a relationship between disability with sex (female), age, frequency of headache and some psychological factors such as depression, catastrophizing and anxiety in patients with headache (15-18). However, all of these studies have focused on migraine and they have ignored the other types of headaches such as tension type headache and cluster headache. In addition, some of the previous studies included headache patients based only on the selfreport and not on clinical examination, which might affect their findings (19). Moreover, no previous study concurrently assessed various variables such as demographic, pain-related and psychological variables in patients with headache. Whereas simultaneous study of these variables leads to comparing their significance in predicting the disability of patients with headache, which may consequently improve pain management of these patients. Finally, most of the previous studies have only studied the patients with chronic or acute headache. Thus, the effect of pain chronicity on disability in patients with headache and its comparison with other variables such as pain intensity, frequency and psychological variables have not been studied. Therefore, the current study was conducted in order to examine the predictive factors of disability in patients with acute and chronic headache.

\section{Objectives}

This study investigated two main issues of research. First, we compared various types of headaches regarding demographic, pain-related and psychological variables. Second, we examined which variables were predictive of disability in patients with headache. We assessed predictive factors of disability based on demographic variables (sex, age, marital status, educational level and employment status), pain-related variables (headache intensity, frequency, chronicity, headache type) and psychological variables (anxiety and depression) in patients with acute and chronic headache.

\section{Methods}

\subsection{Design and Sample}

This cross-sectional study was carried out from January 2017 to July 2018. The target population was the patients with acute or chronic headache (migraine, tension, and cluster) who referred to clinics affiliated with Shiraz University of Medical Sciences. The sample size was determined by NCSS software based on type 1 error (0.05), power (80\%) and effect size (0.19). It was calculated to be 320 patients based on previous studies (20). Due to random sampling problems in this group of patients, including the problems of providing a comprehensive list of patients with headache, a convenience sampling was done within 18 months. Inclusion criteria were as follows: having more than 18 years of age, tendency of involvement in the study and suffering from acute or chronic primary headache based on the diagnosis of a neurologist according to the International Headache Association criteria (9). All the patients were interviewed and examined physically and neurologically by an expert neurologist. During the interview, detailed data were collected regarding the clinical symptoms and features of the headache. Patients were excluded if they had been diagnosed with secondary headaches such as headache due to brain tumors, or with other types of pain such as back pain or if diagnosed with chronic physical or psychological illnesses such as diabetes, schizophrenia and Alzheimer's.

The researcher explained the study to patients. After giving informed consent, the patients received self-report questionnaires. The self-report instruments were completed by patients and returned to the researcher. The data were collected anonymously without name lists. Ethical approval was obtained from the Ethics Committee of the Shiraz University of Medical Sciences.

\subsection{Measures}

In addition to the standard socio-demographic assessment (age, sex, education, employment and marital status), the following measures were used:

\subsubsection{Migraine Disability Assessment Scale}

The migraine disability assessment scale (MIDAS) assesses headache-related disability over a 3-month period. It consists of 7 items. Items 1 and 2 assess the restrictions which are related to school or workplace activities. Items 
3 and 4 assess the restrictions which are related to household work and item 5 assesses the restrictions which are related to social activity or spending leisure time with family members over the past 3 months. Responses are in terms of number of missed days. The total score was obtained by the sum of responses to these 5 questions. Two additional questions are asked about frequency of headache and average pain intensity associated with headaches during the past 3 months. These two last questions are not used in deriving the MIDAS score. In a study by Zandifar et al. (21), the construct validity of the Persian version of the MIDAS was assessed by correlating its total score with the SF-36 mental and physical scores $(r=-0.41, r=-0.36)$ and the pain catastrophizing scale $(r=-0.36)$ in a sample of patients with migraine and tension type headache. The Persian version of scale demonstrated good internal consistency (Cronbach's alpha $=0.82)(21)$. This instrument has shown a high internal consistency (Cronbach's alpha $=0.90$ ) in the present sample.

\subsubsection{Patient Health Questionnaire}

The patient health questionnaire (PHQ) consists of nine items assessing the nine DSM-V (American Psychiatric Association) criteria (22) for major depressive disorder. Each item is rated on a 4-point Likert scale ranging from 'not at all $=0$ ' to 'nearly every day $=3$ '. The total score can range from 0 to 27. Khamseh et al., (23), showed a proper reliability (Cronbach's alpha $=0.87$ ) and criterion validity for the Persian version of the questionnaire. The Persian version showed a high sensitivity (73.8\%) and specificity (76.2\%) for diagnosis of depressive disorder (23). This instrument has proper internal consistency (Cronbach's alpha $=0.87)$ in the current sample.

\subsubsection{Pain Anxiety Symptoms Scale}

The pain anxiety symptoms scale (PASS) is a self-report questionnaire including 20 questions rated on a six-point Likert scale, ranging from never (rated 0) to always (rated 5 ). The Persian version of this tool showed good internal consistency (Cronbach's alpha $=0.86)(24)$. In a study by Paknejad et al. (24), the construct validity of the Persian version of the PASS was assessed by correlating its total score with the pain catastrophizing scale $(r=0.81)$ and the DASS-21 $(r=0.61)$ in a sample of injured workers. This scale had a good internal consistency (Cronbach's alpha $=0.87$ ) in our study.

\subsection{Statistical Analysis}

The gathered data were analyzed by SPSS V. 22 software. The quantitative data were expressed as mean and standard deviation (SD) and qualitative data were expressed as frequency and percentage. Chi-square tests, one-way analyses of variance (ANOVAs) and post hoc Tukey's tests were used for comparing various types of headaches regarding demographic, pain-related and psychological variables. Univariate regression analyses assessed the relation of every potential predictor (independent variable) individually with disability. Independent variables in the univariate regression models included demographic variables (age, sex, marital status, educational level and employment status), pain-related variables (headache intensity, frequency, chronicity, headache type) and psychological variables (anxiety and depression). After that, the variables with a significant correlation with disability were entered into hierarchical multiple regression analyses (method: Enter). First, demographic variables of patients were tested regarding their association with disability. In a second step, pain-related variables, and finally, psychological variables were entered into the model.

\section{Results}

\subsection{Study Sample}

Of the 320 patients who fulfilled the inclusion criteria, a total of 17 patients had to be excluded from the study because they experienced a malignant disease or some other chronic diseases not related to pain (e.g., Alzheimer's, diabetes). The mean age of the patients was 39.85 years old [standard deviation $(\mathrm{SD})=11.37$ ]. The majority of patients were female (76.4\%), married (69.6\%) and about 31\% had a primary school certificate. Average pain intensity over the past 2 weeks was $9.11(\mathrm{SD}=1.07)$ on an 11-point numeric rating scale (NRS). Out of all 303 patients, 195 (64.4\%) had migraine headache. About $15.8 \%$ had tension type headache and $19.8 \%$ were considered to have cluster headache.

\subsection{Comparison of Different Types of Headache}

Results of ANOVAs showed significant differences between the different types of headache regarding age ( $\mathrm{P}=$ 0.000 , Table 1). The results of post hoc tests revealed significant differences between migraine versus tension type and cluster headaches for age. Results of chi-square test showed a higher percent of women among migraine patients compared to the other headache types $(\mathrm{P}=0.000$, Table 1). Also, the frequency of headache over the past three months in patients with migraine was significantly more than the other headache types $(P=0.000$, Table 1$)$. The percentage of chronic pain among migraine and cluster headaches was significantly higher than those with tension type headache $(\mathrm{P}=0.000$, Table 1$)$. There was no significant difference regarding pain intensity in patients with 
different types of headaches $(\mathrm{P}=0.27)$. However, disability $(\mathrm{P}=0.000)$, depression $(\mathrm{P}=0.000)$ and pain-related anxiety $(P=0.000)$ were significantly higher in patients with migraine than those with tension type and cluster headaches.

\subsection{Results of Univariate Regression Models}

The results of univariate regression models showed that sex $(\beta=0.13, \mathrm{P}=0.02)$, educational level $(\beta=-0.12, \mathrm{P}$ $=0.03)$, type of headache $(\beta=-0.13, \mathrm{P}=0.02)$, headache $\operatorname{chronicity}(\beta=0.24, \mathrm{P}=0.000)$, anxiety $(\beta=0.27, \mathrm{P}=0.000)$ and depression $(\beta=0.35, \mathrm{P}=0.000)$ significantly predicted the disability in patients with various types of headache (Table 2).

\subsection{Results of Hierarchical Multiple Regression Analyses}

Multiple regression analyses were performed in order to assess the contributions of variables entered simultaneously to the prediction of disability. In the first step of the hierarchical regression analysis, sex and educational level were entered into the model. Sex $(\beta=0.11, \mathrm{P}=0.04)$ was a significant predictor in this model, but educational level was not. This model reached a variance explanation of $2 \%$. In the second step, headache type and chronicity were included in the model. Headache chronicity contributed to the prediction of disability in this model $(\beta=$ $0.20, \mathrm{P}=0.001)$, but headache type did not. Sex loses its status as a predictive variable in this model $(\mathrm{P}=0.06)$. This model reached a variance explanation of $8 \%$. Finally, psychological variables anxiety and depression were fed into the model. These variables had significant correlations with disability. Headache chronicity also remained a predictive factor in this model $(\beta=0.13, \mathrm{P}=0.01)$. The inclusion of psychological variables led to an $8 \%$ increase in explained variance, for a total variance of $16 \%$ (Table 3 ).

\section{Discussion}

The current study aimed to compare different types of headache in terms of demographic, pain-related and psychological variables. In addition, predictive factors of disability were examined in patients with acute and chronic headache. The results showed that patients with different types of headache revealed no significant differences regarding pain intensity. Migraine patients showed the most pain frequency and chronicity compared to the other patients. The levels of disability, anxiety and depressive symptoms were also higher in patients with migraine compared to patients suffering from cluster or tension type headache. Pain chronicity, anxiety and depression were significantly associated to disability, explaining $16 \%$ of the variance in disability scores.

Results of the present study did not show a significant difference regarding pain intensity in patients with different types of headache. This finding is consistent with our previous study on German patients with chronic headache which were hospitalized in a pain clinic in Kassel area, Germany (14). It may be due to the fact that all of the patients who refer to clinics for an in-patient or outpatient treatment experience high degrees of pain intensity regardless of their diagnosis or type of pain.

In line with some previous studies, the present study revealed the highest frequency of pain among migraine patients compared to the patients suffering from other types of headache $(25,26)$. The percentage of pain chronicity in migraine was also higher than cluster and tension type headache. This finding is in agreement with the definition of headache chronicity based on the criteria of the International Headache Association. According to this, headache is considered chronic, if a person with headache has headache symptoms for at least 3 months and at least 15 days each month. Therefore, it seems logical that the percentage of pain chronicity in patients with migraine is more than the other patients because of higher frequency of pain in these patients. In addition, disability, anxiety and depression in patients with migraine were higher than the other patients with cluster or tension type headache. Several previous studies have shown more severity of depression (27), anxiety $(27,28)$ and disability (29) in patients with migraine compared to the other types of headache. It seems that because patients with migraine experience more frequency of pain and pain chronicity than those with cluster or tension type headache, they also show more symptoms of depression, anxiety and disability. Zwart et al. (27), and Rist et al. (30), also showed the relationship between frequency of headache with depression and anxiety in patients with migraine. Some studies also found the relationship between depression, anxiety and disability with pain chronicity in patients with migraine $(31,32)$. According to Torkamani et al. (33), since patients experience more negative emotions and feelings during headache attacks, therefore the more headache attacks can increase the severity of depression and anxiety in patients with headache. On the contrary, inducing positive emotions, memories, and thoughts in patients with chronic, disabling pain may reduce all facets of pain in these patients (34).

Results of regression analyses showed a significant association between pain chronicity, anxiety and depression with disability in patients with headache. This may be explained by the fact that a high level of anxiety and fear of pain results in the patient avoiding the daily activities 


\begin{tabular}{|c|c|c|c|c|c|c|}
\hline Headache Type & Migraine (N = 195) & Tension $(N=48)$ & & Cluster $(N=60)$ & $\mathbf{F}(\mathbf{d f}) / \chi^{2}(\mathbf{d f})$ & PValue \\
\hline Age & $44.54 \pm 10.79$ & $32.04 \pm 7.09$ & & $33.62 \pm 6.71$ & $F(2,300)=51.35$ & 0.000 \\
\hline Sex, No. (\%) & & & & & $\chi^{2}(2)=38.73$ & 0.000 \\
\hline Male & $27(13.8)$ & $23(47.9)$ & & $27(45)$ & & \\
\hline Female & $168(86.2)$ & $25(52.1)$ & & $33(55)$ & & \\
\hline Headache frequency, day & $19.34 \pm 9.85$ & $11.79 \pm 5.84$ & & $12.43 \pm 6.54$ & $F(2,300)=23.53$ & 0.000 \\
\hline Headache intensity & $9.06 \pm 1.14$ & $9.33 \pm 0.85$ & & 9.100 .96 & $\mathrm{~F}(2,300)=1.28$ & 0.27 \\
\hline Headache chronicity, No. (\%) & & & & & $\chi^{2}(2)=58.29$ & 0.000 \\
\hline Acute & $45(23.2)$ & $39(81.2)$ & & $17(28.3)$ & & \\
\hline Chronic & $149(76.8)$ & $9(18.8)$ & & $43(71.7)$ & & \\
\hline Disability & $21.01 \pm 7.91$ & $11.33 \pm 7.13$ & & $16.03 \pm 6.79$ & $F(2,300)=35.21$ & 0.000 \\
\hline Anxiety & $52.31 \pm 17.58$ & $30.69 \pm 14.50$ & & $38.95 \pm 17$ & $\mathrm{~F}(2,300)=34.84$ & 0.000 \\
\hline Depression & $23.75 \pm 2.72$ & $10.71 \pm 2.45$ & & $15.93 \pm 1.84$ & $F(2,300)=613.63$ & 0.000 \\
\hline Variables & & B & SEB & $\beta$ & $\mathbf{t}$ & PValue \\
\hline \multicolumn{7}{|l|}{ Demographic variables } \\
\hline Age & & 0.01 & 0.04 & -0.01 & -0.28 & 0.77 \\
\hline Sex & & 2.51 & 1.10 & 0.13 & 2.28 & 0.02 \\
\hline Marital status & & -0.15 & 1.05 & 0.009 & -0.14 & 0.88 \\
\hline Educational level & & -1.02 & 0.47 & -0.12 & -2.14 & 0.03 \\
\hline Employment status & & -0.48 & 0.40 & -0.06 & -1.20 & 0.23 \\
\hline \multicolumn{7}{|l|}{ Pain-related variables } \\
\hline Headache frequency & & -0.09 & 0.05 & -0.10 & -1.83 & 0.06 \\
\hline Headache intensity & & 0.39 & 0.45 & 0.05 & 0.88 & 0.38 \\
\hline Headache chronicity & & 4.38 & 0.99 & 0.24 & 4.42 & 0.000 \\
\hline Headache type & & -1.82 & 0.80 & -0.13 & -2.26 & 0.02 \\
\hline \multicolumn{7}{|l|}{ Psychological variables } \\
\hline Anxiety & & 0.12 & 0.02 & 0.13 & 5.04 & 0.000 \\
\hline Depression & & 0.52 & 0.07 & 0.35 & 6.67 & 0.000 \\
\hline
\end{tabular}

which finally lead to disability in the patient (35). Pain anxiety in patients with headache makes the patient not perform the activities that may lead to a worsening of pain. This avoidance, which is due to the fear of severity of the symptoms, results in a sense of helplessness in the patient. Helplessness can in turn affect an individual's understanding of his/her ability in coping with pain as a secondary assessment and the patient feel that he/she is more weakened than what he/she thinks (36). It also seems that since the presence of depressive symptoms in patients can interfere with daily activities and participation in social activities, it increases the patient's disability (37).

There are some possible limitations of our study that should be considered. First, given the cross-sectional design, our findings do not shed light on causal relationships. Moreover, compared to clinical assessment, the accuracy of self-report measures may be affected by response bias (38). Another limitation of the study is related to the inclusion of a sample of patients from clinics affiliated with Shiraz University of Medical Sciences and, thus, prevents generalization. Prospective research with larger samples, and which assesses a wider array of potential predictive factors is needed. Despite these limitations, we assessed the association of various demographic, pain-related and psychological factors with disability in patients with different types of headache assessed by neu- 
Table 3. Prediction of Disability: Results of Hierarchical Regression Analyses Regression Model

\begin{tabular}{|c|c|c|c|c|}
\hline \multirow{2}{*}{ Predictors } & \multicolumn{4}{|c|}{ Criterion: Disability } \\
\hline & $\mathbf{R}^{2}$ & B & $\beta$ & PValue \\
\hline Model 1 & 0.02 & & & \\
\hline Sex & & 2.24 & 0.11 & 0.04 \\
\hline Educational level & & -0.89 & -0.10 & 0.06 \\
\hline Model 2 & 0.08 & & & \\
\hline Sex & & 1.98 & 0.10 & 0.06 \\
\hline Educational level & & -0.52 & -0.06 & 0.27 \\
\hline Headache type & & -0.83 & -0.05 & 0.31 \\
\hline Headache chronicity & & 3.68 & 0.20 & 0.001 \\
\hline Model 3 & 0.16 & & & \\
\hline Sex & & 0.16 & 0.008 & 0.88 \\
\hline Educational level & & -0.35 & -0.04 & 0.45 \\
\hline Headache type & & -0.33 & -0.02 & 0.67 \\
\hline Headache chronicity & & 2.45 & 0.13 & 0.01 \\
\hline Anxiety & & 0.07 & 0.16 & 0.007 \\
\hline Depression & & 0.32 & 0.21 & 0.002 \\
\hline
\end{tabular}

rologists which might be relevant to the management of patients with headache.

\subsection{Conclusions}

In summary, based on our results, pain chronicity, depression and anxiety were associated with disability in patients with headache. The results add some evidence to further support the influence of psychological variables on disability. Our data support cognitive-behavioral models of pain, suggesting an important role for pain-related thoughts and emotions in disability of headache patients. The current findings are relevant to the management of patients with headache. Clinical interventions which reduce anxiety and depression in patients with headache can be an important step in reducing their disability.

\section{Acknowledgments}

The authors thank the patients for their active contribution and participation in this research.

\section{Footnotes}

Authors' Contribution: All authors assisted in the study design. Sasan Rahmanian assisted in the acquisition of data. Farhad Emadi assisted in the patient selection. Maryam Shaygan analysed the data and wrote the first draft of the manuscript. All authors have read and approved the paper.

Conflict of Interests: The authors declare no conflict of interests.

Ethical Approval: Ethical approval was obtained from the Ethics Committee of the Shiraz University of Medical Sciences (IR.SUMS.REC.1396.S589).

Funding/Support: This study was funded by the Shiraz University of Medical Sciences.

Patient Consent: Informed consent was obtained from all participants included in the study.

\section{References}

1. Tollisen A, Selvaag AM, Aulie HA, Lilleby V, Aasland A, Lerdal A, et al. Physical functioning, pain, and health-related quality of life in adults with juvenile idiopathic arthritis: A longitudinal 30-year followup study. Arthritis Care Res (Hoboken). 2018;70(5):741-9. doi: 10.1002/acr.23327. [PubMed: 28732134].

2. Inoue S, Kobayashi F, Nishihara M, Arai YC, Ikemoto T, Kawai T, et al. Chronic pain in the Japanese community-prevalence, characteristics and impact on quality of life. PLoS One. 2015;10(6). e0129262. doi: 10.1371/journal.pone.0129262. [PubMed: 26076135]. [PubMed Central: PMC4467865].

3. International Association for the Study of Pain Subcommittee on Taxonomy. Classification of chronic pain: Descriptions of chronic pain syndromes and definitions of pain terms. 2nd ed. Seatle: IASP press; 1994.

4. Treede RD, Rief W, Barke A, Aziz Q, Bennett MI, Benoliel R, et al. A classification of chronic pain for ICD-11. Pain. 2015;156(6):1003-7. doi: 10.1097/j.pain.0000000000000160. [PubMed: 25844555]. [PubMed Central: PMC4450869].

5. Pahim LS, Menezes AM, Lima R. [Prevalence and factors associated to migraine in adult population, Southern Brazil]. Rev Saude Publica. 2006;40(4):692-8. Portuguese. doi: 10.1590/s003489102006000500020. [PubMed: 17063247].

6. Shahbeigi S, Fereshtehnejad SM, Mohammadi N, Golmakani MM, Tadayyon S, Jalilzadeh G, et al. Epidemiology of headaches in Tehran urban area: A population-based cross-sectional study in district 8, year 2010. Neurol Sci. 2013;34(7):1157-66. doi: 10.1007/s10072-012-12000. [PubMed: 23010877].

7. Kurt S, Kaplan Y. Epidemiological and clinical characteristics of headache in university students. Clin Neurol Neurosurg. 2008;110(1):46-50. doi: 10.1016/j.clineuro.2007.09.001. [PubMed: 17949895].

8. Fattahzadeh-Ardalani G, Sadeghieh Ahari S, Amani F, Moghaddamnia V. Prevalence of migraine and tension headaches and related factors, 2014. Int J Res Med Sci. 2017;5(5):2016. doi: 10.18203/23206012.ijrms20171835

9. Headache Classification Committee of the International Headache Society. Classification and diagnostic criteria for headache disorders, cranial neuralgias and facial pain. Cephalalgia. 1988;8 Suppl 7:1-96. [PubMed: 3048700].

10. Saylor D, Steiner TJ. The global burden of headache. Semin Neurol. 2018;38(2):182-90. doi: 10.1055/s-0038-1646946. [PubMed: 29791944].

11. Smitherman TA, Burch R, Sheikh H, Loder E. The prevalence, impact, and treatment of migraine and severe headaches in the United States: A review of statistics from national surveillance studies. Headache. 2013;53(3):427-36. doi: 10.1111/head.12074. [PubMed: 23470015].

12. Radtke A, Neuhauser H. Prevalence and burden of headache and migraine in Germany. Headache. 2009;49(1):79-89. doi: 10.1111/j.15264610.2008.01263.x. [PubMed: 19125877]. 
13. Leonardi M, Steiner TJ, Scher AT, Lipton RB. The global burden of migraine: Measuring disability in headache disorders with WHO's classification of functioning, disability and health (ICF). J Headache Pain. 2005;6(6):429-40. doi: 10.1007/s10194-005-0252-4. [PubMed: 16388337]. [PubMed Central: PMC3452308].

14. Shaygan M. [Intensity of depression, its predictive and mediating factors in the patients with chronic headache]. Sci J Kurdistan Univ Med Sci. 2017;22(2):110-9. Persian. doi:10.22102/22.2.110.

15. Keogh E, McCracken LM, Eccleston C. Gender moderates the association between depression and disability in chronic pain patients. Eur J Pain. 2006;10(5):413-22. doi: 10.1016/j.ejpain.2005.05.007. [PubMed: 16009583].

16. Mortazavi-Nasiri FS, Pakdaman S, Dehghani M. [The association of catastrophizing and pain-related anxiety with headache-related disability in patients suffering from migraine]. I Res Behav Sci. 2015;13(4):609-16. Persian.

17. Rossi P, Di Lorenzo G, Malpezzi MG, Di Lorenzo C, Cesarino F, Faroni J, et al. Depressive symptoms and insecure attachment as predictors of disability in a clinical population of patients with episodic and chronic migraine. Headache. 2005;45(5):561-70. doi: 10.1111/j.15264610.2005.05110.x. [PubMed: 15953275].

18. Ford S, Calhoun A, Kahn K, Mann J, Finkel A. Predictors of disability in migraineurs referred to a tertiary clinic: neck pain, headache characteristics, and coping behaviors. Headache. 2008;48(4):523-8. doi: 10.1111/j.1526-4610.2008.00859.x. [PubMed: 18377378].

19. Nash JM, Williams DM, Nicholson R, Trask PC. The contribution of pain-related anxiety to disability from headache. J Behav Med. 2006;29(1):61-7. doi: 10.1007/s10865-005-9033-4. [PubMed: 16397822].

20. Chitsaz A, Ghorbani A. [Study of relative distribution of depression and anxiety in suffering from migraine and tension type headache and comparison with those who are not suffering from headache]. $Q$ J Fundam Mental Health. 2005;7(25-26):41-6. Persian.

21. Zandifar A, Asgari F, Haghdoost F, Masjedi SS, Manouchehri N, Banihashemi $\mathrm{M}$, et al. Reliability and validity of the migraine disability assessment scale among migraine and tension type headache in Iranian patients. Biomed Res Int. 2014;2014:978064. doi: 10.1155/2014/978064. [PubMed: 24527462]. [PubMed Central: PMC3914354].

22. American Psychiatric Association. Diagnostic and statistical manual of mental disorders (DSM-5 TM). 5th ed. United States: APA; 2013.

23. Khamseh ME, Baradaran HR, Javanbakht A, Mirghorbani M, Yadollahi Z, Malek M. Comparison of the CES-D and PHQ-9 depression scales in people with type 2 diabetes in Tehran, Iran. BMC Psychiatry. 2011;11:61. doi: 10.1186/1471-244X-11-61. [PubMed: 21496289]. [PubMed Central: PMC3102614].

24. Paknejad M, Asghari A, Rahiminezhad A, Rostami R, Taheri A. [Factorial structure and psychometric properties of the Pain Anxiety Symptoms Scale (PASS-20)]. Appl Psychol Res Q. 2014;5(3):71-93. Persian.

25. Gupta R, Bhatia MS. Comparison of clinical characteristics of migraine and tension type headache. Indian J Psychiatry. 2011;53(2):134-9. doi: 10.4103/0019-5545.82538. [PubMed: 21772645]. [PubMed Central: PMC3136015].
26. Fuh JL, Wang SJ, Lu SR, Liao YC, Chen SP, Yang CY. Headache disability among adolescents: A student population-based study. Headache. 2010;50(2):210-8. doi: 10.1111/j.1526-4610.2009.01531.x. [PubMed: 19804389].

27. Zwart JA, Dyb G, Hagen K, Odegard KJ, Dahl AA, Bovim G, et al. Depression and anxiety disorders associated with headache frequency. The nord-trondelag health study. EurJ Neurol. 2003;10(2):147-52. [PubMed: 12603289].

28. Unalp A, Dirik E, Kurul S. Prevalence and clinical findings of migraine and tension-type headache in adolescents. Pediatr Int. 2007;49(6):943-9. doi: 10.1111/j.1442-200X.2007.02484.x. [PubMed: 18045302].

29. Bera SC, Khandelwal SK, Sood M, Goyal V. A comparative study of psychiatric comorbidity, quality of life and disability in patients with migraine and tension type headache. Neurol India. 2014;62(5):516-20. doi: 10.4103/0028-3886.144445. [PubMed: 25387621].

30. Rist PM, Schurks M, Buring JE, Kurth T. Migraine, headache, and the risk of depression: Prospective cohort study. Cephalalgia. 2013;33(12):1017-25. doi: 10.1177/0333102413483930. [PubMed: 23588795]. [PubMed Central: PMC3720737].

31. Kim SY, Park SP. The role of headache chronicity among predictors contributing to quality of life in patients with migraine: A hospitalbased study. J Headache Pain. 2014;15:68. doi: 10.1186/1129-2377-15-68. [PubMed: 25278151]. [PubMed Central: PMC4194437].

32. Blumenfeld AM, Varon SF, Wilcox TK, Buse DC, Kawata AK, Manack A, et al. Disability, HRQoL and resource use among chronic and episodic migraineurs: Results from the International Burden of Migraine Study (IBMS). Cephalalgia. 2011;31(3):301-15. doi: 10.1177/0333102410381145. [PubMed: 20813784].

33. Torkamani M, Ernst L, Cheung LS, Lambru G, Matharu M, Jahanshahi M. The neuropsychology of cluster headache: cognition, mood, disability, and quality of life of patients with chronic and episodic cluster headache. Headache. 2015;55(2):287-300. doi: 10.1111/head.12486. [PubMed: 25688646]. [PubMed Central: PMC4354253].

34. Shaygan M, Böger A, Kröner-Herwig B. Valence and arousal value of visual stimuli and their role in the mitigation of chronic pain: What is the power of pictures? J Pain. 2017;18(2):124-31. doi: 10.1016/j.jpain.2016.10.007. [PubMed: 27816764].

35. Linton SJ. A review of psychological risk factors in back and neck pain. Spine (Phila Pa 1976). 2000;25(9):1148-56. [PubMed: 10788861].

36. Severeijns R, Vlaeyen JW, van den Hout MA. Do we need a communal coping model of pain catastrophizing? An alternative explanation. Pain. 2004;111(3):226-9. doi: 10.1016/j.pain.2004.07.024. [PubMed: 15363864].

37. Shaygan M, Böger A. [Predictive factors of reducing disability in multidisciplinary treatment of chronic pain].J Mazandaran Univ Med Sci. 2019;28(169):65-74. Persian.

38. Shaygan M, Boger A, Kröner-Herwig B. Neuropathic sensory symptoms: association with pain and psychological factors. Neuropsychiatr Dis Treat. 2014;10:897-906. doi: 10.2147/NDT.S61492. [PubMed: 24899808]. [PubMed Central: PMC4038419]. 